\title{
Effects of painful stimulation and acupuncture on attention networks in healthy subjects
}

\author{
Gang Liu', Hui-juan Ma', Pan-pan Hu', Yang-hua Tian', Shen Hu', Jin Fan²,3 and Kai Wang ${ }^{1 *}$
}

\begin{abstract}
Pain is a subjective sensory and emotional experience, and it has been reported that many different brain regions are regulated by pain, and that pain can impact attention. Acupuncture is an important treatment component of Chinese traditional medicine, and has been used for thousands of years to treat a wide variety of conditions. Although several studies have shown that acupuncture improves consciousness, the precise impact of both acupuncture and painful stimulation on attention is unclear. Are all of the attention networks modulated, or do these stimuli act on a specific network? Is the effect of painful stimulation similar to that of acupuncture? We administered the attention network test to 30 participants (15 males) to investigate the relative efficiencies of three independent attention networks (alerting, orienting, and executive control networks) under three conditions: baseline, after painful stimulation, and after acupuncture. The degree of pain experienced was assessed on a horizontally oriented visual analogue scale. The results showed that painful stimulation and acupuncture had similar effects on the orienting and executive control networks; however, there was a significantly different effect between the three conditions on the alerting network. In conclusion, (1) painful stimulation can selectively impact attention; (2) acupuncture can also selectively impact attention; i.e., both have selective influences on the alerting and executive control networks, but not on the orienting network; (3) the effects of acupuncture and painful stimulation are not identical. The mechanisms by which painful stimulation and acupuncture influence attention warrant further research.
\end{abstract}

Keywords: Painful stimulation, Acupuncture, Attention networks, Alerting network, Orienting network, Executive control network

\section{Introduction}

Pain

Pain is one of the most studied topics in neuroscience, and an almost ubiquitous symptom in a clinical setting. Pain has been defined as "an unpleasant sensory and emotional experience associated with actual or potential tissue damage, or described in terms of such damage" by the International Association for the Study of Pain [1]. It signals danger, and urges action to avoid further damage. When the body is hurt, for example pricked, cut, squeezed, burned, or frozen, nociceptors in the periphery respond, and the central nervous system acts to avoid or alleviate injuries. The properties of pain are related to the underlying

\footnotetext{
*Correspondence: Wangkai1964@126.com

'Department of Neurology, The First Hospital of Anhui Medical University, Hefei, Anhui Province, P. R. China

Full list of author information is available at the end of the article
}

pathological process, with its location generally indicates where the damage has occurred.

Research has demonstrated the involvement of both the peripheral and central nervous systems in pain processing. Pain involves a multifactorial, multipathway system, and each component is thought to be regulated by different brain regions. Human brain imaging studies have identified nociceptive nerve fibers passing through the nuclei of the thalamus, and projecting to higher cortical areas via two main pathways-the lateral and the medial. The lateral pain pathway travels through the ventral posterolateral nucleus and the ventral posteromedial nucleus of the thalamus, and then projects to the primary and secondary somatosensory cortices. The medial pain pathway travels through more medial cortical areas, including the anterior cingulate cortex (ACC), insula, amygdala, and hypothalamus. These two pathways are highly integrated, and both are indispensable for normal pain perception. 
Moreover, these areas are thought to be involved in sensory, motor, emotional, memory, and attentional processes.

\section{Pain and attention}

It is widely known that pain serves as a warning mechanism of danger to the individual: it interrupts, distracts, and demands attention. Moreover, attention deficit is one of the most frequently reported accompanying symptoms in people experiencing either acute or chronic pain.

Many clinical studies have found that pain impacts attentional processes [2], and the brain regions where attention is modulated by pain have been identified by functional magnetic resonance imaging [3]; the ACC and thalamus are known to play key roles in both pain perception and attentional tasks, and pain and attention can both activate the same specific brain areas [4]. However, it remains to be determined whether people experiencing pain have deficits in global attention, or in a specific attention network.

\section{Attention networks}

On the basis of many neuroanatomical and neuropsychological studies, Posner divided the human attention system into three independent networks: the alerting, orienting, and executive control networks. These networks have been distinguished at both the biochemical and cognitive levels, and have distinct neuroanatomical correlates [5-8]. The alerting network is involved in the individual's ability to tonically maintain an alert state, and in producing a phasic response to a warning signal. Its function is critical for optimal performance. It is localized to the thalamus and frontal and parietal areas of the right hemisphere, and primarily utilizes the norepinephrine system. The orienting network is associated with selectively focusing on one, or a few items, out of many candidates. Previous studies have revealed that the orienting network consists of the temporal-parietal junction, superior and inferior parietal lobe, frontal eye fields, and the pulvinar and reticular nuclei of the thalamus, and is modulated by the cholinergic system. Finally, the executive control network refers to the ability to monitor and resolve conflicts in the presence of competing information. Neuroimaging and neuropathological studies have revealed that the ACC and lateral prefrontal cortex are involved in executive control [9-16].

The attention network test (ANT) can simultaneously measure the activity of all three networks, and evaluate their interrelationships [17], and all test results can be obtained within $20 \mathrm{~min}$. Therefore, the ANT has been widely used to investigate attentional function both in healthy subjects, and in patients with disorders such as schizophrenia, Alzheimer's disease, and borderline personality disorder, among others [18-25].

\section{Acupuncture and attention}

Acupuncture is an ancient eastern healing methodology, which is effective in the treatment of various brain disorders, such as psychiatric diseases [26,27], insomnia [28], addiction $[29,30]$, and stroke [31,32]. A review of the literature suggests that acupuncture can indeed influence brain activity [33-35], and modulation of several cortical and subcortical regions, including the insula, amygdala, ACC, and thalamus has been reported [36]. These regions are also highly involved in the limbic system, which plays a significant role in regulating and integrating cognition, emotion, sensory perception, and behavior. However, the neural mechanisms activated by acupuncture are extremely complicated and incompletely understood; in general, acupuncture modulates endogenous regulatory systems, including the autonomic nervous system, the endocrine system, and the neuroendocrine system, and exerts its effects not only through local reflexes but also through the central nervous system [37].

The impact of acupuncture on attention, and its three independent networks, is also unclear. Acupuncture is sometimes accompanied by the sensation of pain; however, compared to painful stimulation, acupuncture appears to have a more complex neural mechanism. Is the effect of painful stimulation similar to that of acupuncture? Do painful stimulation and acupuncture modulate all of the three attention networks, or only one or two specific networks? The present study aimed to investigate and compare the impact of painful stimulation and acupuncture on attention using the ANT. We hypothesize that: (1) painful stimulation can impact attention; (2) acupuncture can impact attention; (3) the effects of acupuncture and painful stimulation on attention are similar.

\section{Methods \\ Subjects}

In order to reduce intersubject variability, participants consisted of a homogeneous group of 30 college students [15 male, 15 females, aged $23.5 \pm 1.2$ (mean $\pm \mathrm{SD}$ ) years] In order to exclude any effects of practice, we recruited another 30 college students [15 male, 15 female, aged $22.3 \pm$ 1.4 (mean $\pm \mathrm{SD}$ ) years] as a control group to perform the ANT three times in a similar environment and on a similar schedule. All subjects were recruited from Anhui Medical University located in Hefei City, Anhui Province, China. All were right handed, and acupuncture naive. They all had normal speaking, writing, language expression, and cognitive skills. None had any history of serious physical disorders or of mental illness. Our study protocol was approved by the Anhui Medical University Ethics Committee, and written informed consent was obtained from all participants before the study. 


\section{Procedures and measures}

The experiment was divided into three blocks: baseline, after painful stimulation, and after acupuncture. During each block, all participants were administered the same ANT task. To exclude any interference of painful stimulation and acupuncture on the baseline block, each experiment began with the baseline block. Then, all participants were re-administered the ANT, immediately after either painful stimulation or after acupuncture. Lastly, the ANT was re-administered immediately after the remaining stimulus condition. In order to reduce possible influences on the results, participants were not informed of the order in which painful stimulation and acupuncture blocks would be performed, and in order to facilitate blinding, they were instructed to keep their eyes closed to prevent them from actually observing the procedures, and were asked to remain relaxed without engaging in any mental tasks. The sequence of painful stimulation and acupuncture was counterbalanced across all participants. There was a 5-min rest period after each ANT (Figure 1).

\section{Baseline condition}

Unlike the painful stimulation and acupuncture blocks, during the baseline condition, all participants were administered the ANT task without any interruption. We designed the baseline block as the control condition.

\section{Painful stimulation condition}

The acupuncture needles (sterile disposable stainless steel acupuncture needle, $0.3 \mathrm{~mm}$ in diameter and $40 \mathrm{~mm}$ in length) were inserted in 2 non-meridian points $(2-3 \mathrm{~cm}$ away from ST36 [Stomach 36, Zusanli] near bilateral ST36 acupoints). The needles were inserted vertically, to a depth of $30 \mathrm{~mm}$ (Table 1). Subjects felt pain, but reported no feelings of soreness, fullness, heaviness or numbness. The needle remained at rest for $2 \mathrm{~min}$ (R1) before bidirectional rotation at $1 \mathrm{~Hz}$ for $2 \mathrm{~min}$ (M1). The needle was not manipulated for $3 \mathrm{~min}$ (R2), and then, manipulation was repeated for $2 \mathrm{~min}$ (M2), followed by

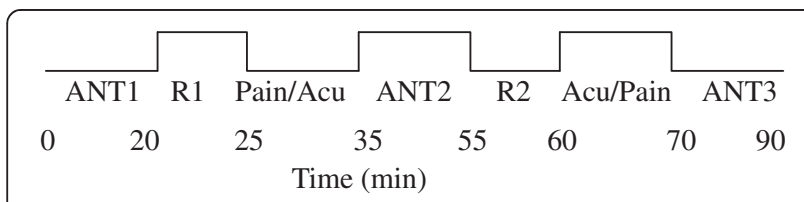

Figure 1 Time course of the entire experimental procedure. Each participant was administered the ANT three times: at baseline, after painful stimulation, and after acupuncture. First, all participants were administered the ANT1 without any interruption (Baseline). Then, all were re-administered the ANT2 immediately after painful stimulation (Pain) or after acupuncture (Acu). Lastly, all were re-administered the ANT3 immediately after acupuncture (Acu) or after painful stimulation (Pain). The sequence of Pain and Acu were counterbalanced across all participants. There was a 5-min rest period (R1, R2) after each ANT. a third period of rest for $1 \mathrm{~min}$ (R3), then the needle was removed (Figure 2).

The entire procedure took $10 \mathrm{~min}$. We used the horizontally oriented visual analogue scale (VAS) to assess the degree of pain experienced [38,39], with the mean VAS score being $5.23 \pm 0.49$ (mean $\pm \mathrm{SD}$ ) (Figure 3 ).

\section{Acupuncture condition}

The acupuncture needles (sterile disposable stainless steel acupuncture needle, $0.3 \mathrm{~mm}$ in diameter and $40 \mathrm{~mm}$ in length) were inserted in 2 ST36 acupoints (5 mm below the head of the fibula under the knee joint, and $2 \mathrm{~mm}$ lateral to the anterior tubercle of the tibia) (see Figure 2A). The needles were inserted vertically, to a depth of $30 \mathrm{~mm}$. The subjects felt "De-qi" sensations, such as soreness, fullness, heaviness, and numbness. The manipulation and procedure were identical to that applied in the painful stimulation condition (see Figure 2B). To exclude the feeling of dull or sharp pain, we again used the VAS to assess the degree of pain experienced during acupuncture, with the mean VAS score being $1.24 \pm 0.27$ (mean \pm SD). This was significantly different from the painful stimulation condition ( $\mathrm{T}=35.877, P<0.001)$. The procedure was performed by the same experienced and licensed acupuncturist on all subjects.

\section{Control group}

In order to exclude the effects of practice from the experiment, we recruited another 30 college students as a control group, to perform the ANT three times in a similar environment and on a similar schedule.

\section{Between-subject comparison}

In order to clarify whether there were any potential carryover effects between the painful stimulation and acupuncture conditions, we conducted a comparison between the 15 subjects who performed pain stimulation test first, and the 15 subjects who performed the pain stimulation test after acupuncture. We also compared the 15 subjects who performed the acupuncture test first, and the 15 subjects who performed the acupuncture test after pain stimulation.

\section{Attention network test}

The attention network test was created using E-Prime (Version 1.1, Psychology Software Tools, Pittsburgh, PA, USA). Participants viewed the stimuli shown on a computer screen, and responses were automatically collected via two response buttons. The stimuli consisted of a row of five horizontal black lines, with arrowheads pointing left or right, and the target was a left or right pointing arrowhead in the center, against a gray background. The target stimulus was flanked on either side by two arrows pointing either in the same direction (congruent condition), 
Table 1 Procedure used for the painful stimulation and acupuncture conditions

\begin{tabular}{lll}
\hline & \multicolumn{1}{c}{ Painful stimulation } & \multicolumn{1}{c}{ Acupuncture } \\
\hline Location & non-meridian points $(2-3 \mathrm{~cm}$ away from ST36) & ST36 \\
Needle gauge & $0.3 \mathrm{~mm}$ in diameter and $40 \mathrm{~mm}$ in length & $0.3 \mathrm{~mm}$ in diameter and $40 \mathrm{~mm}$ in length \\
Needle insertion depth & under the skin $30 \mathrm{~mm}$ & under the skin $30 \mathrm{~mm}$ \\
Insert direction & vertically & vertically \\
\hline
\end{tabular}

or in the opposite direction (incongruent condition), or by nothing (neutral condition). Participants were instructed to focus on a centrally located stationary cross throughout the task, and to respond as quickly and accurately as possible. The participant's task was to identify the direction of the center arrow by pressing one button for the left direction with the index finger of their left hand, or a second button for the right direction with the index finger of their right hand. The target stimulus remained on the screen until the participant responded, but the maximum response time was cut off at $1700 \mathrm{~ms}$. Cues consisted of an asterisk appearing for $100 \mathrm{~ms}$, presented $400 \mathrm{~ms}$ before the presentation of the target. There were four cue conditions in the process: (1) no cue, the participant was shown a cross at the same location as the first stationary cross for $100 \mathrm{~ms}$; (2) a center cue, an asterisk was presented on the central point; (3) a double cue, an asterisk was presented at two target locations simultaneously, above and below the central point; and (4) a spatial cue, an asterisk was presented at a target location either above or below the central point. The whole process of the ANT consisted of a 24-trial practice block, and three experimental blocks of trials. The presentation of trials was in a random order for each participant. Each experimental block consisted of 96 trials (48 different conditions: 4 cue types $\times 2$ target locations $\times 2$ target directions $\times 3$ congruencies, with 2 repetitions). The entire ANT was completed in 20 min (Figure 4).

\section{Calculation of attention network efficiencies}

The ANT makes use of differences in reaction times (RTs) derived from the different experimental conditions to measure the efficiency of the alerting, orienting, and executive control networks [17]. Alerting efficiency was calculated by subtracting the mean RTs of the conditions with double cues from those of the conditions with no cue, as neither of these conditions provided any information on the spatial location of the target. Similarly, orienting efficiency was calculated by subtracting the mean RTs of the conditions with spatial cues from those of the conditions with center cues. In both conditions, the subject was alert, but only the spatial cue provided orientation information. Likewise, executive control efficiency was calculated by subtracting the mean RTs of congruent target conditions from those of incongruent target conditions.
A

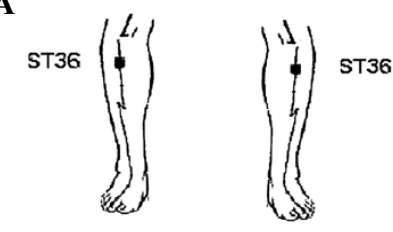

B

needle in needle out

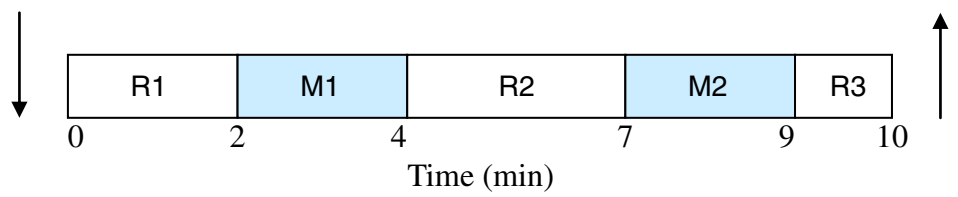

Figure 2 Painful stimulation and acupuncture conditions: A ST36: $5 \mathrm{~mm}$ below the head of the fibula under the knee joint, and $2 \mathrm{~mm}$ lateral to the anterior tubercle of the tibia. $\mathbf{B}$ shows the paradigm for painful stimulation and acupuncture: The needle remained at rest for 2 min (R1) before bidirectional rotation at $1 \mathrm{~Hz}$ for $2 \mathrm{~min}$ (M1). The needle was not manipulated for $3 \mathrm{~min}$ (R2), and then, manipulation was repeated for 2 min (M2), followed by a third period of rest for 1 min (R3). Then the needle was removed. 


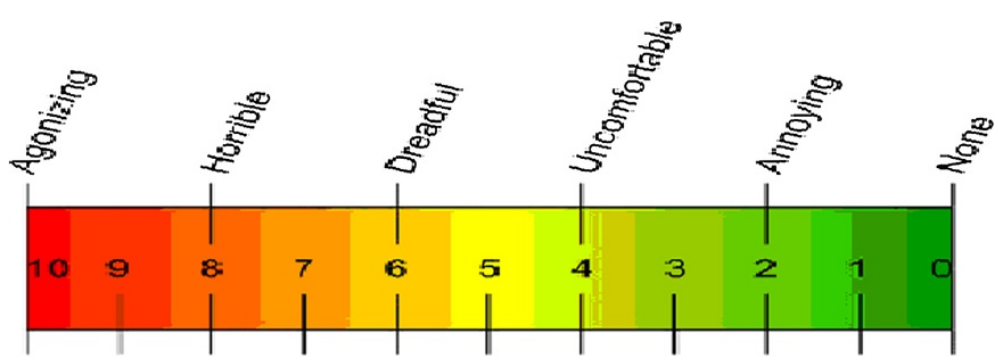

Figure 3 Visual Analogue Scale of pain (VAS). VAS is presented as a ruler with a movable cursor, the length being approximately $10 \mathrm{~cm}$. "0" represents "No pain," while "10" represents "Agonizing." When the test began, subjects moved the cursor to the position on the ruler that best represented the degree of pain they were currently experiencing.

\section{Data analysis}

Statistical analysis was performed using SPSS (Version 13.0, Statistical Program for Social Sciences, SPSS Inc., Chicago, IL, USA). The statistical significance of differences between the baseline, after painful stimulation, and after acupuncture conditions was evaluated by a one-way analysis of variance (ANOVA); the betweensubject comparison was evaluated using an independent samples t-test, and the threshold for statistical significance was set at $P<0.05$. To assess differences between individual conditions, a Student-Newman-Keuls (SNK) test was also used.

(a) Cue conditions

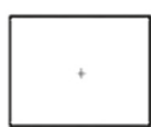

no cuc

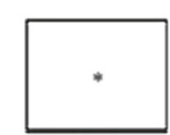

center cue

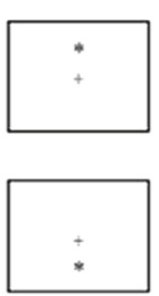

spatial cue

(b) Target conditions

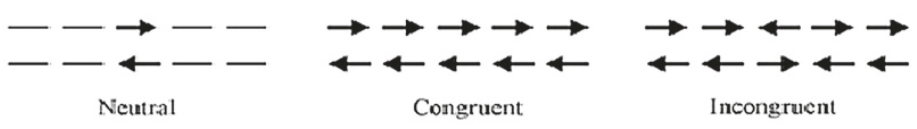

(c) Presentation time course

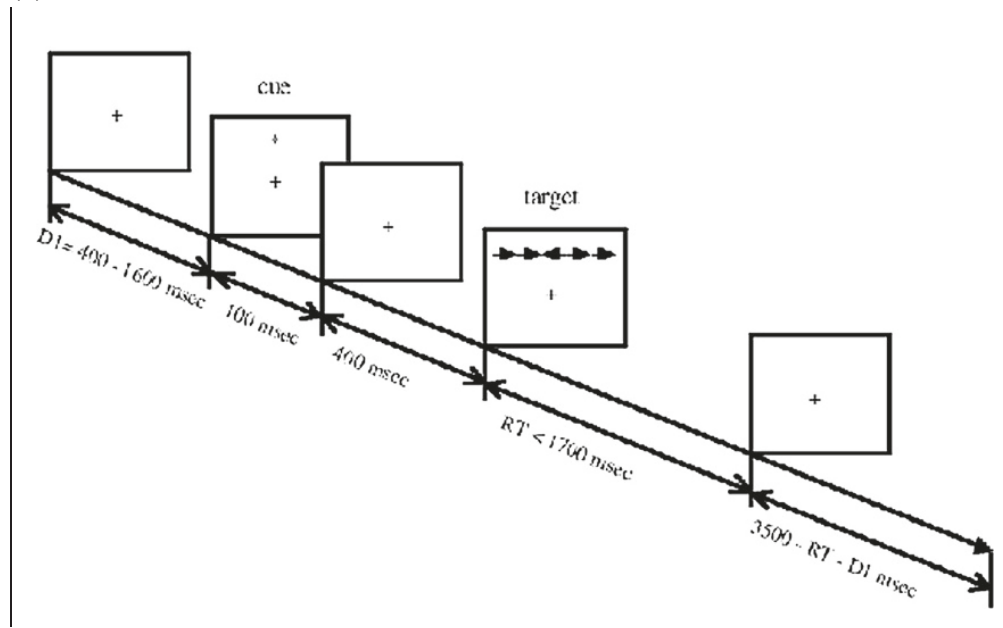

Figure 4 Experimental paradigm for the attention network test. (a) The four cue conditions. (b) The six stimuli used in the present experiment. (c) An example of the procedure and the time course of a trial using a spatial cue with incongruent flankers. 


\section{Results}

Reaction times and accuracy

The mean RTs and accuracy rates for each of the three conditions are summarized in Table 2. During the experiment, any incorrect or missed responses were excluded from the data set. We conducted a 4 (cue condition: center cue, double cue, no cue, spatial cue) $\times 3$ (flanker type: congruent, incongruent, neutral) repeated measures ANOVA on the RT data listed in Table 2. There was a significant main effect for both cue condition and flanker type ([i] baseline: cue condition, F $(3,87)=147.225, P<0.001$; flanker type, $\mathrm{F}(2,58)=345.498, P<0.001$; interaction of cue conditions $\times$ flanker types, $F(6,174)=8.626$, $P<0.001$; [ii] after painful stimulation: cue condition, $\mathrm{F}(3,87)=167.751, P<0.001$; flanker type, $\mathrm{F}(2,58)=$ 382.327, $P<0.001$; interaction of cue condition $\times$ flanker type, $\mathrm{F}(6,174)=18.639, P<0.001$; and [iii] after acupuncture: cue condition, $\mathrm{F}(3,87)=250.158, P<0.001$; flanker type, $F(2,58)=415.649, P<0.001$; interaction of cue condition $\times$ flanker type, $\mathrm{F}(6,174)=20.530, P<0.001)$.

We conducted an analysis of variance with repeated measures for accuracy as well, but there were no significant main effects of cue conditions or flanker type in the three conditions (Table 2).

\section{Effects of painful stimulation and acupuncture on the three attention networks}

The effects of the three attention networks in three conditions are summarized in Table 3 . There was a significant main effect of the three conditions on the alerting network tasks $(\mathrm{F}(2,87)=9.200, P<0.001)$. Our results show that participants were significantly more vigilant following painful stimulation or acupuncture than in the baseline condition (SNK, $P<0.05$ ), and the comparison between painful stimulation and acupuncture conditions revealed a significant difference (SNK, $P<0.05$ ). There was a significant main effect of the three conditions on the executive control network tasks $(\mathrm{F}(2,87)=8.811$, $P<0.01)$. Participants had less difficulties in resolving conflict after either painful stimulation or acupuncture than in the baseline condition (SNK, $P<0.05$ ), while there was no significant difference between the painful stimulation and acupuncture conditions (SNK, $P>0.05$ ). However, there were no significant differences between the three conditions on the orienting network tasks $(\mathrm{F}(2,87)=2.398, P>0.05)$. Thus, participants exhibit specific improvements in the performance of the alerting and executive control networks after either painful stimulation or acupuncture, but there was no impact on the function of the orienting network. There also was a significant main effect of the three conditions on the overall mean RTs $(\mathrm{F}(2,87)=23.238, P<0.01)$. Participants took significantly less time to finish the test following either painful stimulation or acupuncture than they did in the baseline condition (SNK, $P<0.05$ ). There was no significant difference between overall mean RTs after either of the two interferences (SNK, $P>0.05$ ). Additionally, there was no significant difference observed in response accuracy between the three conditions $(F(2,87)=0.811$, $P>0.05$ ) (Figure 5).

\section{Correlation between the VAS and the attention networks}

We examined the relationship between the VAS and the scores of the three attentional network tests. No significant correlations were identified between the VAS and the three network scores for either the painful stimulation or the acupuncture condition ([i] the painful stimulation: VAS and alerting, $r=0.218, P>0.05$; VAS and orienting, $r=0.097, P>0.05$; VAS and executive control, $r=0.273, P>0.05$; [ii] the acupuncture condition: VAS and alerting, $r=0.065, P>0.05$; VAS and orienting, $r=0.071, P>0.05$; VAS and executive control, $r=$ $-0.125, P>0.05)$.

\section{Control group results}

There were no significant differences observed in alerting, orienting, and executive control networks between the three trials performed by the control group ([i] Alerting: $\mathrm{F}(2,87)=0.070, P>0.05$; [ii] Orienting: $\mathrm{F}(2,87)=$ $0.361, P>0.05$; [iii] Executive control: $F(2,87)=0.168$, $P>0.05)$

\section{Between-subject comparison results}

There were no significant differences observed in the alerting, orienting, or executive control networks in a between-subject comparison of the pain stimulation and the acupuncture conditions ([i] pain stimulation: Alerting, $\mathrm{T}=0.364, P>0.05$; Orienting, $\mathrm{T}=0.348, P>0.05$; Executive control, $\mathrm{T}=0.071, P>0.05$ ); [ii] acupuncture condition: Alerting, $\mathrm{T}=0.006, P>0.05$; Orienting, $\mathrm{T}=0.921$, $P>0.05$; Executive control, $\mathrm{T}=0.795, P>0.05)$.

\section{Discussion}

Our study used the ANT to measure participant's performance under baseline conditions, after painful stimulation, and after acupuncture, in order to investigate how painful stimulation and acupuncture might impact the three distinct networks of attention. Painful stimulation was used as an independent variable, and the subjective experience of pain as measured by VAS was significantly different between the painful stimulation and acupuncture conditions. No significant correlations were identified between VAS and the three network scores for either the painful stimulation or the acupuncture conditions. Our experimental results exclude any significant effects of practice on the outcome, and confirmed there were no carryover effects between the painful stimulation and acupuncture conditions. We found that there were 
Table 2 Mean reaction times and accuracy under each cue condition for baseline, after painful stimulation, and after acupuncture

\begin{tabular}{|c|c|c|c|c|c|c|c|c|c|c|c|c|}
\hline \multirow[t]{2}{*}{ Condition } & \multicolumn{3}{|c|}{ No cue } & \multicolumn{3}{|c|}{ Double cue } & \multicolumn{3}{|c|}{ Center cue } & \multicolumn{3}{|c|}{ Spatial cue } \\
\hline & Congruent & Incongruent & Neutral & Congruent & Incongruent & Neutral & Congruent & Incongruent & Neutral & Congruent & Incongruent & Neutral \\
\hline \multicolumn{13}{|c|}{ Mean RTs (ms) and standard deviations } \\
\hline Baseline & $609(82)$ & $715(93)$ & $563(69)$ & $570(74)$ & $697(87)$ & $521(66)$ & $575(77)$ & $706(90)$ & $531(69)$ & $543(72)$ & $640(86)$ & $493(59)$ \\
\hline After painful stimulation & $593(75)$ & $686(87)$ & $557(61)$ & $554(69)$ & $664(86)$ & $497(63)$ & $5560(75)$ & $673(85)$ & $500(64)$ & $524(74)$ & $608(93)$ & $476(62)$ \\
\hline After acupuncture & $592(82)$ & $686(84)$ & $553(72)$ & $545(76)$ & $656(89)$ & $486(64)$ & $547(76)$ & $664(91)$ & $493(71)$ & $516(77)$ & $597(91)$ & $469(62)$ \\
\hline \multicolumn{13}{|c|}{ ACC and standard deviations } \\
\hline Baseline & $0.99(0.01)$ & $0.97(0.04)$ & $0.99(0.01)$ & $0.99(0.01)$ & $0.98(0.03)$ & $1.00(0.00)$ & $0.99(0.01)$ & $0.97(0.05)$ & $0.99(0.02)$ & $1.00(0.00)$ & $0.98(0.03)$ & $0.99(0.01)$ \\
\hline After painful stimulation & $0.99(0.01)$ & $0.97(0.05)$ & $0.99(0.01)$ & $0.99(0.01)$ & $0.98(0.03)$ & $1.00(0.00)$ & $1.00(0.00)$ & $0.97(0.06)$ & $0.99(0.01)$ & $0.99(0.01)$ & $0.99(0.02)$ & $0.99(0.01)$ \\
\hline After acupuncture & $0.99(0.01)$ & $0.96(0.06)$ & $0.99(0.01)$ & $0.99(0.01)$ & $0.98(0.03)$ & $0.99(0.00)$ & $1.00(0.00)$ & $0.96(0.07)$ & $0.99(0.01)$ & $0.99(0.01)$ & $0.99(0.02)$ & $1.00(0.00)$ \\
\hline
\end{tabular}


Table 3 Attention network scores (in RT and ratio score) of baseline, after painful stimulation, and after acupuncture

\begin{tabular}{|c|c|c|c|c|c|c|}
\hline & & & After & tion & Aft & ure \\
\hline & Mean & SE & Mean & SE & Mean & SE \\
\hline Alerting (ms) RT & 29.43 & 3.73 & 37.27 & 3.66 & 45.47 & 3.54 \\
\hline Ratio & 0.05 & 0.01 & 0.07 & 0.01 & 0.08 & 0.01 \\
\hline Orienting (ms) RT & 45.8 & 3.59 & 38.83 & 3.34 & 40.6 & 2.46 \\
\hline Ratio & 0.08 & 0.01 & 0.07 & 0.01 & 0.07 & 0.00 \\
\hline Executive control (ms) RT & 116.17 & 6.57 & 100.8 & 5.93 & 99.73 & 5.02 \\
\hline Ratio & 0.2 & 0.01 & 0.18 & 0.01 & 0.18 & 0.01 \\
\hline Accuracy (\%) & 98.63 & 0.2 & 98.8 & 0.24 & 98.6 & 0.26 \\
\hline Overall mean (ms) RT & 594.8 & 13.36 & 572.37 & 13.16 & 565.1 & 13.58 \\
\hline
\end{tabular}

significant effects of both pain and acupuncture stimuli on the alerting network, as well as on the executive control network. In contrast, no effect of either was found on the orienting network. Moreover, a participant's performance demonstrated that acupuncture increased alertness to a greater extent than did painful stimulation. These results suggest that painful stimulation and acupuncture both exert effects on the alerting and executive control attention networks, but not on the orienting network; they also indicate that, in the alerting network, the effect of acupuncture is quantitatively different from that of painful stimulation.

Several previous studies have shown that pain influences cognitive abilities [40-42]. As expected, the present

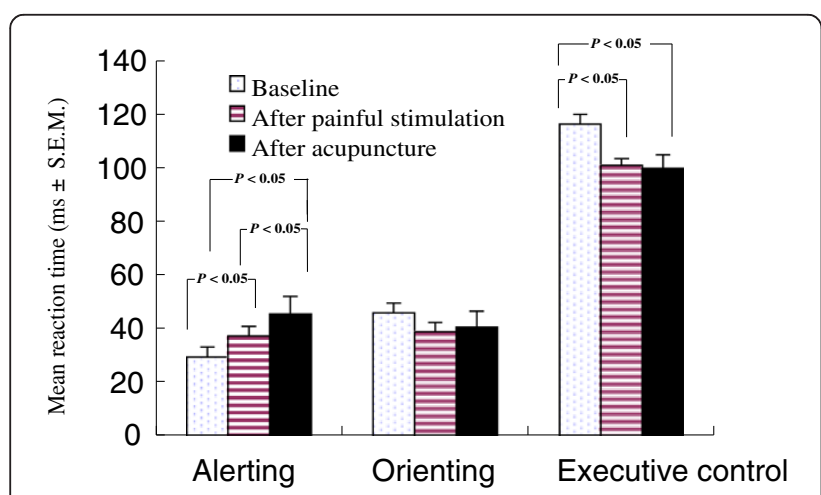

Figure 5 Selective modulation of the three attention networks after the painful stimulation and acupuncture conditions. Bar chart shows means and standard errors. The results show that with regards to alerting, participants were significantly more vigilant after either the painful stimulation or acupuncture conditions than in the baseline condition (SNK, $P<0.05)$, and the comparison between the painful stimulation and acupuncture conditions also showed a significant difference (SNK, $P<0.05$ ). With regards to executive control, participants had less difficulties in resolving conflict after either painful stimulation or acupuncture than under baseline conditions ( $\mathrm{SNK}, P<0.05)$, and there was no significant difference between the painful stimulation and acupuncture conditions (SNK, $P>0.05$ ). With regards to orienting, there was no significant difference in performance between any of the three conditions (SNK, $P>0.05$ ). study showed that alerting functions increased after the administration of either painful stimulation or acupuncture, with participants becoming more vigilant than they were under baseline condition. These stimuli may place them in a vigilant state, thereby ensuring that they are capable of reacting to any warning signal that may require an immediate response. Some patients with chronic pain have indeed exhibited an attentional bias, or hypervigilance for painful stimuli, which is believed to play a role in the development and maintenance of chronic pain states $[43,44]$. Several authors have found that an association exists between pain and hypervigilance $[45,46]$. One such study found that subjects showed an increased level of attention in a semantic task, which consisted of either word generation (category fluency) or word repetition, while they were receiving a painful stimulus [47].

Neural activity in several brain regions is increased in painful conditions as compared to pain-free conditions, and many imaging studies have demonstrated a minimal involvement of the prefrontal cortex, and particularly the orbitofrontal cortex and thalamus [48]. As we know, the alerting network is localized to the thalamus, frontal and parietal areas of the right hemisphere, involves the cortical projections of the norepinephrine system [49], and is responsible for activating and maintaining a vigilant state. When faced with pain, the body comes into a state of emergency, with an increase in norepinephrine secretion, which might lead to excessive norepinephrine levels in the prefrontal cortex. This might induce hypervigilance after pain. Alternatively, one study suggested that people have a limited capacity for attention, with pain being an extremely powerful noxious stimulus that demands attention, decreasing the resources available to perform other cognitive functions [50].

In our study, the performance of the executive control network was significantly decreased after both the painful stimulation and acupuncture conditions. Consistent with our first and second hypotheses, our results indicate that participants resolve conflicts more rapidly after either painful stimulation and acupuncture than they do 
under baseline conditions. As part of attentional processes, the executive control network is responsible for monitoring and resolving conflicts. On the basis of neuroanatomical and neurotransmitter research, the executive control network has been localized to the midline frontal areas, such as the ACC and prefrontal cortex, is modulated by dopamine (DA), and acts to monitor and resolve conflicts between competing information. Previous studies on animals and humans have shown that the medial frontal cortex, and particularly the ACC, can greatly reinforce acute nociceptive responses; for example, formalin injections enhanced behavior with disgust experience $[51,52]$. Furthermore, patients with frontal lesions or cingulate resections often cannot feel pain [53]. Electroencephalographic and neuroimaging studies have revealed that there are specific nociceptive neurons in the ACC that respond to noxious stimuli [54-59]. Additionally, lesions to dopaminergic neurons [60] located in the prefrontal cortex [61] result in the impairment of the executive control network. One study suggested that an age-related improvement in the performance of executive control tasks is paralleled by changing expression levels of DA and its receptor or gene [62].

In our study, the orienting network was not affected by either painful stimulation or acupuncture. This is in agreement with the findings of several other studies. For example, no difference was found in the function of the orienting network from 6 years of age to adulthood, the findings indicating that the orienting network as assessed by the ANT remains stable during brain development [21], and children with idiopathic generalized epilepsy had no deficit in the orienting network either [63]. However, some authors have suggested that exogenous orienting would be enhanced when the cue was painful [64]. In contrast to this, it has been shown that neuronal responses to nociceptive stimuli are weaker and less effective than responses to anti-nociceptive stimuli in orienting attention [65]. In our present study, the orienting network was not affected by either painful stimulation or acupuncture, but its mechanisms are not well understood, and warrant further research in the future.

In our present study, the results confirmed that acupuncture can impact selective attention networks, enhancing the efficiency of the alerting and executive control networks; notably, when compared to painful stimulation, acupuncture had a significantly greater effect on the alerting network. This is contrary to our third hypothesis; however, the mechanisms underlying this difference have yet to be elucidated. While the neural mechanisms of acupuncture are unknown, it has been suggested that it may induce an increased release of endorphins, serotonin, norepinephrine, or $\gamma$-aminobutyric acid [66]. Acupuncture might improve cognitive function by influencing neurotransmitter levels. The exact interaction between painful stimulation and acupuncture warrant further research in the future.

\section{Limitations}

There are a number of potential limitations to the current study. We included a 5-min rest period after each ANT; it is possible that this period was not long enough for subjects to rest well. Future studies could include a longer rest period between trials.

In conclusion, the results of the current investigation support the existence of selective effects on the alerting and executive control attentional networks following either painful stimulation or acupuncture. To the best of our knowledge, this is the first study to concurrently assess the effects of both painful stimulation and acupuncture on the three attention networks, using the ANT. From a clinical/functional point of view, we confirmed that: (1) painful stimulation can impact attention; (2) acupuncture can impact attention; (3) the effects of acupuncture and painful stimulation are not identical. Further studies could combine the ANT with functional magnetic resonance imaging and positron emission computed tomography in order to more precisely investigate the neural mechanisms underlying the influence of painful stimulation and acupuncture on functional attention networks.

\section{Competing interest}

The authors have declared that they have no competing interest.

\section{Authors' contributions}

Conceived and designed the experiments: GL, JF, KW. Performed the experiments: GL, HJM, SH, YHT. Analyzed the data: GL, PPH. Wrote the paper: $\mathrm{GL}$. All authors read and approved the final manuscript.

\section{Financial disclosure}

This work was supported by a grant from the National Natural Science Foundation of China (Grant \# 81171273), and the National Basic Research Program of China (973 Program) (Grant \#2011CB707805).

The funding organizations had no role in the study design, data collection, data analysis, data interpretation, or writing of the report. The corresponding author had full access to all of the data in the study and takes responsibility for the integrity of the data and the accuracy of the data analysis.

\section{Author details}

${ }^{1}$ Department of Neurology, The First Hospital of Anhui Medical University, Hefei, Anhui Province, P. R. China. '2Department of Psychology, Queens College, City University of New York, New York, NY 10029, USA.

${ }^{3}$ Departments of Psychiatry and Neuroscience, Mount Sinai School of Medicine, New York, NY 10029, USA.

Received: 2 October 2012 Accepted: 2 June 2013

Published: 7 June 2013

\section{References}

1. Eccleston C: Chronic pain and attention: a cognitive approach. $\mathrm{Br} \mathrm{J}$ Clin Psychol 1994, 33(Pt 4):535-547.

2. Dick BD, Rashiq S: Disruption of attention and working memory traces in individuals with chronic pain. Anesth Analg 2007, 104:1223-1229. tables of contents.

3. Buffington AL, Hanlon CA, McKeown MJ: Acute and persistent pain modulation of attention-related anterior cingulate $\mathrm{fMRI}$ activations. Pain 2005, 113:172-184. 
4. Bantick SJ, Wise RG, Ploghaus A, Clare S, Smith SM, Tracey I: Imaging how attention modulates pain in humans using functional MRI. Brain 2002, 125(Pt 2):310-319.

5. Posner Ml, Petersen SE: The attention system of the human brain. Annu Rev Neurosci 1990, 13:25-42.

6. Posner MI, DiGirolamo GJ, Fernandez-Duque D: Brain Mechanisms of Cognitive Skills. Conscious Cogn 1997, 6:267-290.

7. Posner Ml, Gilbert CD: Attention and primary visual cortex. Proc Natl Acad Sci USA 1999, 96:2585-2587.

8. Fossella J, Posner MI, Fan J, Swanson JM, Pfaff DW: Attentional phenotypes for the analysis of higher mental function. ScientificWorldJournal 2002, 2:217-223.

9. Marrocco RT, Davidson MC: Neurochemistry of attention. In The Attentive Brain. Edited by Parasuraman R. Cambridge, Mass: MIT; 1998:35-50.

10. Corbetta M, Shulman GL: Control of goal-directed and stimulus-driven attention in the brain. Nat Rev Neurosci 2002, 3:201-215.

11. Benes FM: Emerging principles of altered neural circuitry in schizophrenia. Brain Res Brain Res Rev 2000, 31(2-3):251-269.

12. Fossella J, Sommer T, Fan J, Wu Y, Swanson JM, Pfaff DW, Posner MI: Assessing the molecular genetics of attention networks. BMC Neurosci 2002, 3:14.

13. Fan J, Fossella J, Sommer T, Wu Y, Posner Ml: Mapping the genetic variation of executive attention onto brain activity. Proc Natl Acad Sci USA 2003, 100:7406-7411.

14. Diamond A, Briand L, Fossella J, Gehlbach L: Genetic and neurochemical modulation of prefrontal cognitive functions in children. Am J Psychiatry 2004, 161:125-132.

15. Thienel R, Kellermann T, Schall U, Voss B, Reske M, Halfter S, Sheldrick AJ, Radenbach K, Habel U, Shah NJ, Kircher T: Muscarinic antagonist effects on executive control of attention. Int J Neuropsychopharmacol 2009, 12:1307-1317.

16. Thienel R, Voss B, Kellermann T, Reske M, Halfter S, Sheldrick AJ, Radenbach K, Habel U, Shah NJ, Schall U, Kircher T: Nicotinic antagonist effects on functional attention networks. Int J Neuropsychopharmacol 2009, 12:1295-1305.

17. Fan J, McCandliss BD, Sommer T, Raz A, Posner Ml: Testing the efficiency and independence of attentional networks. J Cogn Neurosci 2002, 14:340-347.

18. Posner Ml, Rothbart MK, Vizueta N, Levy KN, Evans DE, Thomas KM, Clarkin JF: Attentional mechanisms of borderline personality disorder. Proc Natl Acad Sci USA 2002, 99:16366-16370.

19. Klein RM: Chronometric explorations of disordered minds. Trends Cogn Sci 2003, 7:190-192

20. Mezzacappa E: Alerting, orienting, and executive attention: developmental properties and sociodemographic correlates in an epidemiological sample of young, urban children. Child Dev 2004, 75:1373-1386

21. Rueda MR, Fan J, McCandliss BD, Halparin JD, Gruber DB, Lercari LP, Posner MI: Development of attentional networks in childhood. Neuropsychologia 2004, 42:1029-1040.

22. Sobin C, Kiley-Brabeck K, Daniels S, Blundell M, Anyane-Yeboa K, Karayiorgou M: Networks of attention in children with the $22 q 11$ deletion syndrome. Dev Neuropsychol 2004, 26:611-626.

23. Wang K, Fan J, Dong Y, Wang CQ, Lee TM, Posner Ml: Selective impairment of attentional networks of orienting and executive control in schizophrenia. Schizophr Res 2005, 78:235-241.

24. Fernandez-Duque D, Black SE: Attentional networks in normal aging and Alzheimer's disease. Neuropsychology 2006, 20:133-143.

25. Gu X, Liu X, Guise KG, Fossella J, Wang K, Fan J: Alexithymic trait and voluntary control in healthy adults. PLoS One 2008, 3:e3702.

26. Wang SM, Peloquin C, Kain ZN: The use of auricular acupuncture to reduce preoperative anxiety. Anesth Analg 2001, 93:1178-1180.

27. Wu J, Yeung AS, Schnyer R, Wang Y, Mischoulon D: Acupuncture for depression: a review of clinical applications. Can J Psychiatry 2012, 57:397-405.

28. Yeung WF, Chung KF, Leung YK, Zhang SP, Law AC: Traditional needle acupuncture treatment for insomnia: a systematic review of randomized controlled trials. Sleep Med 2009, 10:694-704.

29. Mayer DJ: Acupuncture: an evidence-based review of the clinical literature. Annu Rev Med 2000, 51:49-63.

30. Ulett GA, Han J, Han S: Traditional and evidence-based acupuncture: history, mechanisms, and present status. South Med J 1998, 91:1115-1120.
31. Zhao XF, Du Y, Liu PG, Wang S: Acupuncture for stroke: evidence of effectiveness, safety, and cost from systematic reviews. Top Stroke Rehabil 2012, 19:226-233.

32. Inoue I, Chen L, Zhou L, Zeng X, Wang H: Reproduction of scalp acupuncture therapy on strokes in the model rats, spontaneous hypertensive rats-stroke prone (SHR-SP). Neurosci Lett 2002, 333:191-194.

33. Esch T, Guarna M, Bianchi E, Zhu W, Stefano GB: Commonalities in the central nervous system's involvement with complementary medical therapies: limbic morphinergic processes. Med Sci Monit 2004, 10:MS6-MS17.

34. Hui KK, Liu J, Marina O, Napadow V, Haselgrove C, Kwong KK, Kennedy DN, Makris N: The integrated response of the human cerebro-cerebellar and limbic systems to acupuncture stimulation at ST 36 as evidenced by fMRI. Neuroimage 2005, 27:479-496.

35. Samuels N, Gropp C, Singer SR, Oberbaum M: Acupuncture for psychiatric illness: a literature review. Behav Med 2008, 34:55-64.

36. Feng Y, Bai L, Ren Y, Wang H, Liu Z, Zhang W, Tian J: Investigation of the large-scale functional brain networks modulated by acupuncture. Magn Reson Imaging 2011, 29:958-965.

37. Hori E, Takamoto K, Urakawa S, Ono T, Nishijo H: Effects of acupuncture on the brain hemodynamics. Auton Neurosci 2010, 157:74-80.

38. Price DD, McGrath PA, Rafii A, Buckingham B: The validation of visual analogue scales as ratio scale measures for chronic and experimental pain. Pain 1983, 17:45-56.

39. Ogon M, Krismer M, Söllner W, Kantner-Rumplmair W, Lampe A: Chronic low back pain measurement with visual analogue scales in different settings. Pain 1996, 64:425-428.

40. Eccleston C, Crombez G, Aldrich S, Stannard C: Attention and somatic awareness in chronic pain. Pain 1997, 72:209-215.

41. Dick B, Eccleston C, Crombez G: Attentional functioning in fibromyalgia, rheumatoid arthritis, and musculoskeletal pain patients. Arthritis Rheum 2002, 47:639-644.

42. Harman K, Ruyak P: Working through the pain: a controlled study of the impact of persistent pain on performing a computer task. Clin J Pain 2005, 21:216-222

43. Beck JG, Freeman JB, Shipherd JC, Hamblen JL, Lackner JM: Specificity of Stroop interference in patients with pain and PTSD. J Abnorm Psychol 2001, 110:536-543.

44. Keogh E, Ellery D, Hunt C, Hannent I: Selective attentional bias for painrelated stimuli amongst pain fearful individuals. Pain 2001, 91:91-100.

45. Roelofs J, Peters ML, McCracken L, Vlaeyen JM: The pain vigilance and awareness questionnaire (PVAQ): further psychometric evaluation in fibromyalgia and other chronic pain syndromes. Pain 2003, 101:299-306.

46. Asmundson GJ, Wright KD, Hadjistavropoulos HD: Hypervigilance and attentional fixedness in chronic musculoskeletal pain: consistency of findings across modified stroop and dot-probe tasks. J Pain 2005, 6:497-506.

47. Rémy F, Frankenstein UN, Mincic A, Tomanek B, Stroman PW: Pain modulates cerebral activity during cognitive performance. Neuroimage 2003, 19:655-664.

48. Apkarian AV, Bushnell MC, Treede RD, Zubieta JK: Human brain mechanisms of pain perception and regulation in health and disease. Eur J Pain 2005, 9:463-484.

49. Coull JT, Nobre AC, Frith CD: The noradrenergic alpha2 agonist clonidine modulates behavioural and neuroanatomical correlates of human attentional orienting and alerting. Cereb Cortex 2001, 11:73-84.

50. Kuhajda MC, Thorn BE, Klinger MR, Rubin NJ: The effect of headache pain on attention (encoding) and memory (recognition). Pain 2002, 97:213-221.

51. Lee DE, Kim SJ, Zhuo M: Comparison of behavioral responses to noxious cold and heat in mice. Brain Res 1999, 845:117-121.

52. Johansen JP, Fields HL, Manning BH: The affective component of pain in rodents: direct evidence for a contribution of the anterior cingulate cortex. Proc Natl Acad Sci USA 2001, 98:8077-8082.

53. Zhuo M: Glutamate receptors and persistent pain: targeting forebrain NR2B subunits. Drug Discov Today 2002, 7:259-267.

54. Talbot JD, Marrett S, Evans AC, Mever E, Bushnell MC, Duncan GH: Multiple representations of pain in human cerebral cortex. Science 1991, 251:1355-1358.

55. Sikes RW, Vogt BA: Nociceptive neurons in area 24 of rabbit cingulate cortex. J Neurophysiol 1992, 68:1720-1732.

56. Rainville P, Duncan GH, Price DD, Carrier B, Bushnell MC: Pain affect encoded in human anterior cingulate but not somatosensory cortex. Science 1997, 277:968-971. 
57. Casey KL: Forebrain mechanisms of nociception and pain: analysis through imaging. Proc Natl Acad Sci USA 1999, 96:7668-7674.

58. Hutchison WD, Davis KD, Lozano AM, Tasker RR, Dostrovsky JO: Pain-related neurons in the human cingulate cortex. Nat Neurosci 1999, 2:403-405.

59. Rainville P, Bushnell MC, Duncan GH: Representation of acute and persistent pain in the human CNS: potential implications for chemical intolerance. Ann N Y Acad Sci 2001, 933:130-141.

60. Simon H, Scatton B, Moal ML: Dopaminergic A10 neurones are involved in cognitive functions. Nature 1980, 286:150-151.

61. Brozoski TJ, Brown RM, Rosvold HE, Goldman PS: Cognitive deficit caused by regional depletion of dopamine in prefrontal cortex of rhesus monkey. Science 1979, 205:929-932.

62. Lidow MS, Goldman-Rakic PS, Gallager DW, Rakic P: Distribution of dopaminergic receptors in the primate cerebral cortex: quantitative autoradiographic analysis using $[3 \mathrm{H}]$ raclopride, $[3 \mathrm{H}]$ spiperone and $[3 \mathrm{H}]$ SCH23390. Neuroscience 1991, 40:657-671.

63. Tian Y, Dong B, Ma J, Zhou S, Zhou N, Wang K: Attention networks in children with idiopathic generalized epilepsy. Epilepsy Behav 2010, 19:513-517.

64. Van Damme S, Crombez G, Lorenz J: Pain draws visual attention to its location: experimental evidence for a threat-related bias. J Pain 2007, 8:976-982.

65. Legrain V, Damme SV, Eccleston C, Davis KD, Seminowicz DA, Crombez G: A neurocognitive model of attention to pain: behavioral and neuroimaging evidence. Pain 2009, 144:230-232.

66. Sun Y, Gan TJ, Dubose JW, Habib AS: Acupuncture and related techniques for postoperative pain: a systematic review of randomized controlled trials. Br J Anaesth 2008, 101:151-160.

doi:10.1186/1744-9081-9-23

Cite this article as: Liu et al:: Effects of painful stimulation and acupuncture on attention networks in healthy subjects. Behavioral and Brain Functions 2013 9:23.

\section{Submit your next manuscript to BioMed Central and take full advantage of:}

- Convenient online submission

- Thorough peer review

- No space constraints or color figure charges

- Immediate publication on acceptance

- Inclusion in PubMed, CAS, Scopus and Google Scholar

- Research which is freely available for redistribution 